\title{
Efficient Power Control for Broadcast in Wireless Communication Systems
}

\author{
A. T. Chronopoulos \\ Computer Science Department \\ University of Texas at San Antonio \\ San Antonio, TX 78249 \\ Email:atc@cs.utsa.edu
}

\author{
P. Cotae \\ Department of Electrical Engineering, \\ University of Texas at San Antonio, \\ San Antonio, TX 78249 \\ Email:pcotae@utsa.edu
}

\author{
S. Ponipireddy \\ Computer Science Department \\ University of Texas at San Antonio \\ San Antonio, TX 78249 \\ Email:sponipir@cs.utsa.edu
}

\begin{abstract}
Energy efficiency is a measure of performance in wireless networks. Therefore, controlling the transmitter power at a given node increases not only the operating life of the battery but also the overall system capacity by successfully admitting new nodes between a source and a destination. It is essential to find effective means of power control of point-to-point, broadcasting and multicasting scenarios. In past work [9], we presented a new scheme State Space-based Control Design (SSCD) using both the state space and optimal control methodology in discrete-time for power control in wireless systems. Further, we proved the convergence of the overall network with our algorithm using Lyapunov stability analysis. We made a comparison with a well known Distributed Power control (DPC) scheme in [3,4]. Here we present simulation results and comparisons for point-to-point communication with random node placement. We also combined the schemes SSCD and DPC with a tree based broadcast algorithm BIP to obtain broadcast tree in ad-hoc networks with power control. We show the effectiveness of the new algorithm through simulations.
\end{abstract}

Key words and Phases: Energy efficiency, Wireless Network, Distributed Power Control.

\section{INTRODUCTION}

In any wireless multiple-access system, the need for power control is evident. The problems of broadcast access in allwireless networks are being currently studied. Several articles study point-to-point and multicast/broadcast communication for example see [1], [2], ,3], [4],[5],[10], ,111, [12]. In [9] we considered the problem of deriving a new distributed power control scheme (SSCD) using state space and optimal control in discrete time. We proved the convergence of the overall network and compared the effectiveness of our method to the DPC method [3] , [4] for uniformly placed nodes. Here we implement DPC and SSCD and make comparisons for randomly placed nodes. The wireless networking environment presents formidable challenges to the above study. Among the most difficult issues related to mobile wireless applications is that of operation in limited-energy environments. In this paper we propose to use distributed power control scheme with one of the main Quality of Service feature that considers 'SIR' for the addition of each node in broadcast tree method. The schemes developed in [1], [9] address the issues of transmitter power levels (and hence network connectivity), and the formation of a link (routing). This approach relies on the 'node-based' nature of wireless communication.
To assess each complex trade-off separately, we assume the following:

- No mobility.

- The availability of a large number of bandwidth resources. (So that contention for channel is not an issue)

- Sufficient transceiver resources are available at each node. (So calls are never blocked)

- The channel conditions are unchanged.

Under these assumptions we focus on the determination of minimum-energy broadcast tree construction taking into consideration the SIR. In the following sections we study the Wireless communication model (Section 2), Power control approaches (Section 3), Minimum energy broadcast tree (Section 4), Broadcast incremental power (BIP) with SIR (Section 5), Simulations (Section 6) and conclusions (Section 7).

\section{A Wireless Communications Model}

In wireless networks it is possible to establish a node between any pair of nodes, provided that each has a transceiver available for this purpose and that the SIR at the receiver node is sufficiently high, (i.e above the required threshold). The nodes in the wireless networks are determined depending on factors such as distance between nodes, transmitter power, error-control schemes, other-user interference, and background noise. Furthermore, in wireless networks no distinction can be made between up-node and down-node traffic. This complicates the interference environment.

The connectivity of the network depends on the transmission power and the interference at the receiver node. Assuming that each node can set its own power level, which is not to exceed a maximum value $P_{\max }$. We assume that the receiver signal power varies as $r^{-\alpha}$, where $r$ is the range and $\alpha$ is a parameter that typically takes on a value between 2 and 4 depending on the characteristics of the communication medium.

\section{Power Control Approaches}

Our goal is to maintain a required SIR threshold for each network node while the transmitter power is adjusted so that the least possible energy is consumed. Suppose there are $n$ nodes in the network. Let $G_{i j}$ be the power loss(gain) from the transmitter of the $i$ th node to the receiver of the $j$ th node. It involves the free space loss, multi-path fading, 
shadowing, and other radio wave propagation effects, as well as the spreading/processing gain of CDMA transmissions [7]. The power attenuation is taken to follow the inverse fourth power law

$$
G_{i i}=\frac{g}{r_{i j}^{\alpha}},
$$

Calculation of SIR $R_{i}$ at $i$ th node, [3]

$$
R_{i}=\frac{G_{i i} * P_{i}}{\left(\Sigma_{j \neq i} G_{i j} * P_{j}+\eta_{i}\right)},
$$

where $i, j \in\{1,2,3, \ldots, n\}, P_{i}$ is the $i^{t h}$ node's transmitter power and $\eta_{i}>0$ is the thermal noise at its receiver node. For each node $i$ there is a lower SIR threshold $\gamma$. We take the threshold to be the same as $\gamma$ for all nodes, reflecting a certain QoS the node has to maintain in order to operate properly. Therefore, we require

$$
R_{i} \geq \gamma
$$

for every $i=1,2,3, . ., n$.

\section{A. Distributed Power Control(DPC):}

The above condition is used to minimize the transmitter power but it is not mandatory. If the equation (3) fails (i.e $\left.R_{i}<\gamma\right)$ then the transmitter power of the transmitter has to be updated using equation (4). Therefore each node independently increases its power when its current SIR is below its target $\gamma$, and decreases it otherwise. The associated power update can be obtained from [4] as

$$
P_{i}(k+1)=\frac{\gamma P_{i}(k)}{R_{i}(k)}
$$

where $k=(1,2,3, .$.$) (see [3] - [5]). If P_{i}(k+1)>P_{\max }$, the new node is not added. If the power slips under the minimum threshold power $P_{i}(k+1)<P_{\min }$ (the minimum power needed to form a link), then we keep $P_{i}(k+1)=P_{\text {min }}$.

SIR protection of an active node

For any active node $i$, that we have $R_{i}(k) \geq \gamma \quad=>$ $R_{i}(k+1) \geq \gamma$. This implies that a new node is added if and only if the new state of the system is stable, i.e. none of the existing nodes are broken. The final state contains all the feasible nodes added out of $n$ nodes maintaining a stable state. Though the work of [4] can be expressed in the above algorithm, but there is strong evidence that the power update can cause convergence problems. We proposed a state space scheme for updating the transmitter power [9] which addresses the limitations of DPC.

\section{B. State Space-based Control Design (SSCD):}

Using state space theory [8] our goal is to maintain a required SIR threshold for each network node while the transmitter power is adjusted so that the least possible energy is consumed. Using (2) the SIR denoted here as, $R_{i}$, at the $(k+1)$ th iteration can be written as

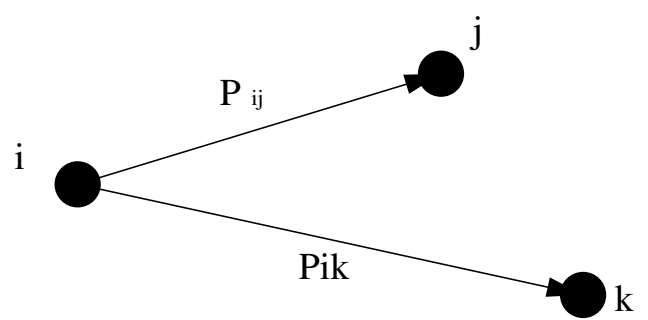

Fig. 1. The "wireless broadcast advantage." $P_{i,(j, k)}=\max \left\{P_{i j}, P_{i k}\right\}$

$$
R_{i}(k+l)=R_{i}(k)+v_{i}(k),
$$

where by definition $R_{i}=P_{i} / I_{i}$, and interference $I_{i}(k)=$ $\left(\sum_{j \neq i}^{n} P_{j} * \frac{G_{i j}}{G_{i i}}+\frac{\eta_{i}}{G_{i i}}\right)$, with $\mathrm{n}$ is the number of active nodes. The $v_{i}$ in each system should only depend on the total interference produced by the other users. To maintain the SIR of each node above a desired target its new power is computed as follows,

$$
P_{i}(k+1)=R_{i}(k+1) I_{i}(k) .
$$

Using this new transmitter power, the SIR's are calculated and checked till the stable state of all the nodes is achieved.

\section{Minimum Energy Broadcast Tree}

The Broadcast Incremental power algorithm (BIP) in [1] determines the minimum- power tree routed at the source node, that reaches all the other nodes in the network. For wireless networks, this is a difficult problem for which no scalable solutions appear to be available. Therefore, this heuristic based algorithm is explained in [1]. This is a node-based (rather than link-based) approach because it enables to exploit the wireless multicast advantage.

\section{Wireless broadcast advantage}

$P_{i j}=$ power needed for link between Node $\mathrm{i}$ and Node $\mathrm{j}$ $=r^{\alpha}$

where $r$ is the distance between node Node $\mathrm{i}$ and Node $\mathrm{j}$.

An example of broadcasting to 2 destination nodes which includes wireless broadcast advantage is shown here. In this simple example, there are two alternative strategies:

1. S transmits using $P_{s 2}$ : both $D_{1}$ and $D_{2}$ anre reached.

2. S transmits using $P_{s 1}$ : only $D_{1}$ is reached.

$D_{1}$ then transmits to $D_{2}$ with power $P_{12}$, resulting in a total power of $P_{s 1}+P_{12}$.

Here, source-initiated, circuit-switched, broadcast sessions have been considered. The network consists of $\mathrm{N}$ nodes, randomly distributed over a specific region. Any node is permitted to initiate broadcast sessions. Each broadcast group consists of a source node. Some of the nodes may act as relays also, either to provide connectivity to all members of the broadcast group or to reduce overall energy consumption or both. These set of nodes that support a broadcast session (the source node, all other nodes with in the range) is referred to as a broadcast tree.

The connectivity of the network depends on the transmission power. Assuming that each node can choose its own power 


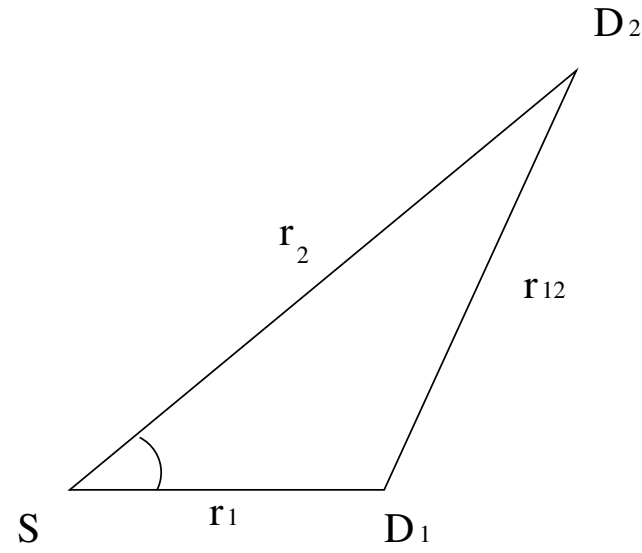

Fig. 2. Broadcasting to two destinations

level, which is not to exceed a maximum value $P_{\max }$. We assume that the receiving signal power varies as $r^{-\alpha}$, where $r$ is the range and $\alpha$ is a parameter that typically takes on a value between 2 and 4 depending on the characteristics of the communication medium. Based on this model the transmitting power required to support a link between two nodes, separated by range $r$, is proportional to $r^{\alpha}$.

The Broadcast Incremental Power (BIP) Algorithm presented in [1] (based on Prim's minimum spanning tree algorithm) [9] is determined to get the minimum-energy tree being routed at the source node that reaches all the other nodes in the network. This algorithm exploits the wireless broadcast advantage in the construction of the broadcast tree, but the QoS issues are not considered in [1]. Here we included one of the main QoS issue i.e. SIR at the receiving nodes and present the following algorithm.

\section{Broadcast InCREMENTAl Power (BIP) WITH SIR}

We next present the distributed algorithm [9] for constructing a broadcast tree with power control. We denote the local source node by SN.

\section{Algorithm:}

1. Each SN receives the position (i.e. coordinates of each node) of a subset of the nodes to be directly linked to it.

2. SN starts the broadcast tree formation according to the BIP algorithm in [9]. Before adding a new link to the tree:

a. Each future receiver node calculates the SIR and checks against its threshold.

b. The new SIR will be sent to SN.

c. SN checks the SIR, if any of the conditions (2) or (3) is not satisfied then the new transmitting powers are calculated according to the equations (4) and(5) respectively.

4. If any new nodes are still unlinked then, Go to step 1 . Else tree construction is complete.

The final tree is the minimum-Energy broadcast tree, for the given set of $N$ nodes.

\section{Simulations}

The setup for the simulation experiments to investigate DPC schemes developed in [4] and our SSCD is as follows. The network region is assumed to span a square region of side 500 units. All receiving nodes have the same SIR target $\gamma=5 \mathrm{dBm}$. The normalized noise floor $\eta_{i} / g$ from equation (2) is taken to be the same for all receivers and equal to $3.973 e^{-17}$. The initial power value for each node is assigned as $P_{0}=-20 \mathrm{dBm}$. We have an upper limit on the power of each transmitter i.e. $P_{\max }=20 \mathrm{dBm}$. When the transmitter power of an active node is in danger of exceeding $P_{\max }$, while a new inactive node is being admitted, the new node is not added to the system. A new node is added only if it maintains the system's stable state even after its addition. The SIR's of all the active nodes are maintained just above the target $\gamma$.

Point to point simultaneous links In the first scenario, we consider 10 point to point links Figure 3 (with a transmitter and a receiver) which are placed randomly. In order to generate a spatially uniform statistical mixture of links, each one is randomly constructed as follows. For every new link, the link transmitter is uniformly placed in the given square region. The link receiver is placed isotropically around its transmitter (given a reference direction, the link angle is distributed in $[0,2 \pi)$ uniformly) and at a random distance from it. In this scenario, we can see that the speed of convergence is faster for SSCD approach in comparison with the DPC approach Figure 4 and 5. In the above run the DPC scheme took more than 35 iterations where as SSCD took less than 35 iterations Figures 4 and 5. One more observation here is, that the fluctuation of SIR's in DPC is more and it often goes below the threshold $(\gamma=5.0)$. In SSCD its minimal. From this we can say that the active links are protected better in SSCD than in the DPC scheme. In terms of system capacity, the SSCD scheme admits more new links than DPC scheme. This clearly shows the superiority of SSCD scheme over the DPC scheme. Also, the total energy of the system is more for SSCD when compared to DPC [4]. This can be explained as a tradeoff between the total energy consumed and the speed of convergence.

Broadcasting In this scenario, a broadcast tree is constructed based on the above algorithm. Shown here Figure 6 is a scenario with 10 nodes randomly placed and node 2 as the source node. The above algorithm is simulated with both the DPC and SSCD approachs. The SIR's of each node are plotted while the tree was forming. Figure 7 shows the DPC approach while Figure 8 shows the SSCD approach. The final tree consumes minimum energy and is stable until a new node arrives. From the figures we can see that DPC converges faster than SSCD approach while the total energy consumed by SSCD approach is less than DPC approach.

\section{CONCLUSIONS}

This paper presents an energy efficient power control scheme for broadcast in wireless networks. We implemented two existing Distributed Power Control schemes (SSCD and DPC). At first, we compare them for randomly placed nodes 


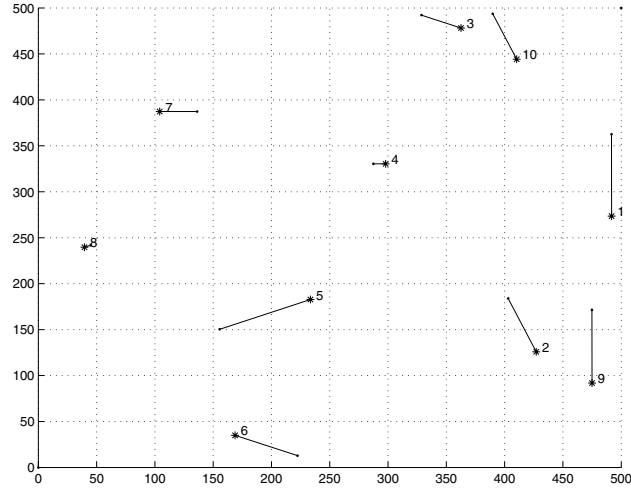

Fig. 3. Random distribution of links

for point to point communication. Then we include these two schemes as part of the new algorithm for broadcast communication. We run simulations to study the feasibility of the new broadcast algorithm and compared the control schemes as part of this algorithm.

\section{ACKNOWLEDGMENTS}

(1) NSF CCR-0312323 (2003-2006), (2) NASA NAG21383 (1999-2001), (3) State of Texas Higher Education Coordinating Board through the Texas Advanced Research/Advanced Technology Program ATP 003658-04421999

\section{REFERENCES}

[1] A. Ephremides, J. E. Wieselthier and G. D. Nguyen, "On the Construction of Energy-Efficient Broadcast and Multicast Trees in Wireless Networks". pp.585-594, IEEE INFOCOM 2000.

[2] M. H. Ammar, G. C. Polyzos and S. K. Tripathi, "Special Issue on network support for multipoint communication" IEEE Journal on Selected Areas in Communications, 15, April 1997.

[3] N. Bambos, "Towards Power-Sensitive Network Architectures in Wireless Communications:concepts, issues and Design Aspects" . IEEE Personal Communications, pp.50-59, June 1998.

[4] N. Bambos, S. Chen and G. J. Pottie, "Channel Access Algorithms with Active Link protection for Wireless Communication Networks with Power Control". IEEE ACM Transactions on networking, pp.583-597, October 2000.

[5] G. J. Foschini and Z. Miljanic, "A Simple Distributed autonomous power control algorithm and its convergence". IEEE Tran. Veh. Tech., vol.42, pp.641-646, April 1993.

[6] A. El-Osery, and C. Abdallah "Distributed Power Control in CDMA Cellular Systems", IEEE Antennas and Propagation Magazine, Vol. 42, No.4, August 2000.

[7] T. S. Rappanport, "Wireless Communications, Principles and Practices", book Prentice Hall, 1999.

[8] F.L. Lewis, "Optimal Control", John Wiley and Sons, 1999.

[9] A. T. Chronopoulos, J. Sarangapani, S. Ponipireddy, "Distributed Power Control in Wireless Communication Systems", at the IEEE International Conference on Computer Communications and Networks (ICCCN 2002), Oct. 2002, pp 493-496.

[10] N. Herscovici, C. Christodoulou, A. El-Osery, and C. Abdallah "Distributed Power Control in CDMA Cellular Systems", IEEE Antennas and Propagation Magazine, Vol. 42, No.4, August 2000.

[11] C. Tang and C. S. Raghavendra, "Energy Efficient Adaptation of Multicast Protocol in Power Controlled Wireless Ad Hoc Networks", International Symposium on Parallel Architectures, Algorithms and Networks (ISPAN '02) May, 2002

[12] K. Obraczka, G. Tsudik "Multicast Routing Issues in Ad Hoc Networks", IEEE 1998.

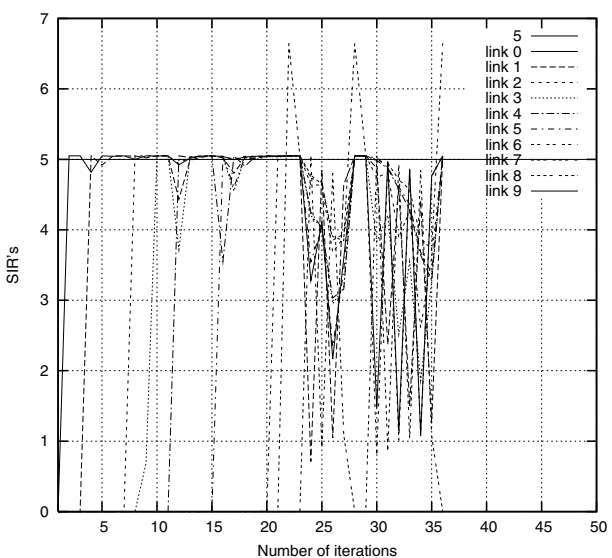

Fig. 4. DPC with Random link

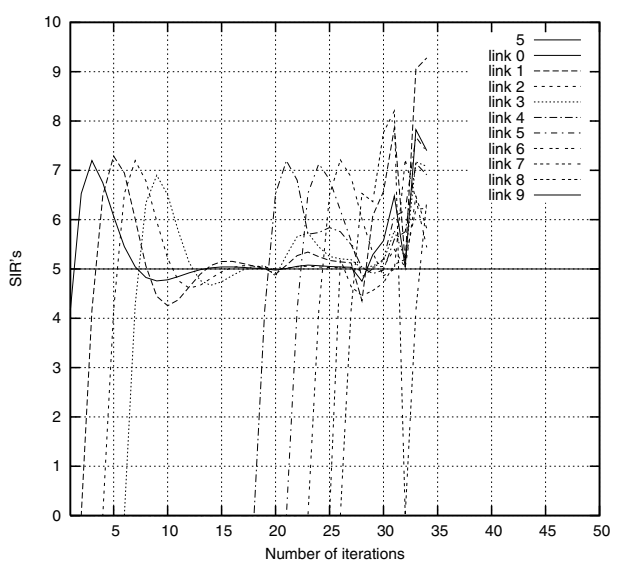

Fig. 5. SSCD with Random link

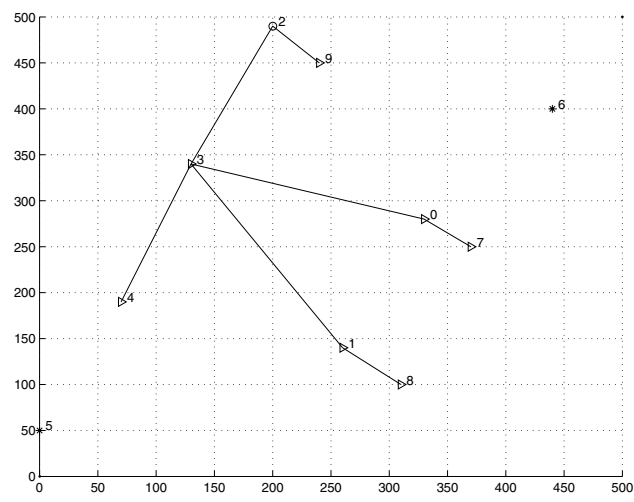

Fig. 6. Converged Broadcast Tree 


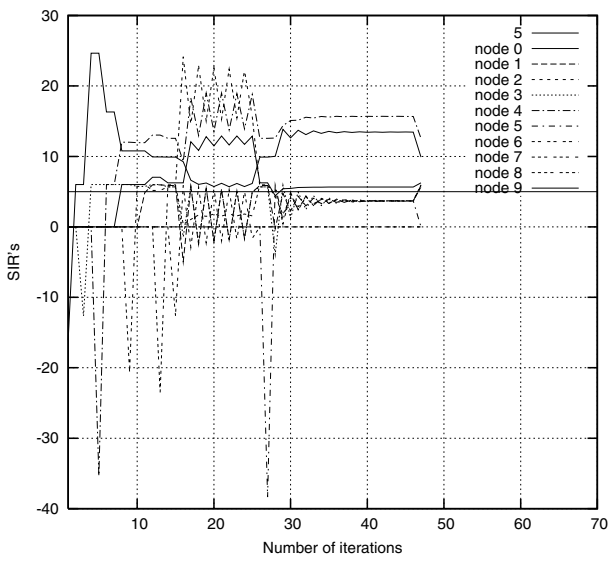

Fig. 7. DPC

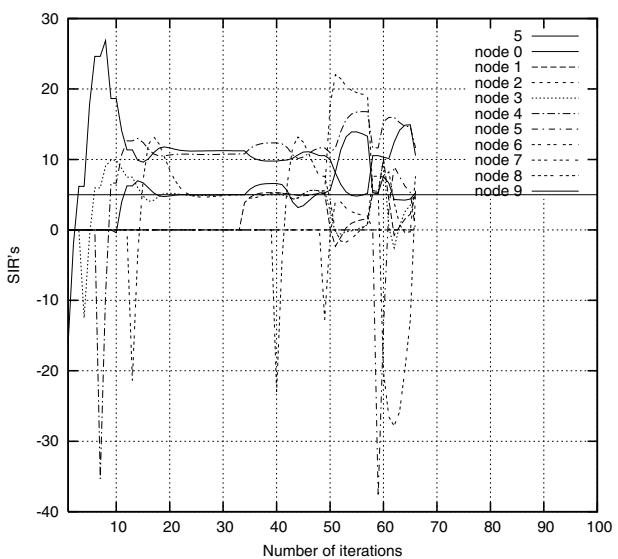

Fig. 8. SSCD 